\title{
Intracranial tuberculoma and recent advances in magnetic resonance imaging
}

\author{
Ahmad A. Moussa ${ }^{1,2^{*}}$, Mohamed E. Mahmoud ${ }^{1}$ and Hosam A. Yousef ${ }^{3}$
}

\begin{abstract}
Background: Tuberculosis is resurging both in the developing and developed worlds. This includes TB of the central nervous system which is more common in children. It carries a high mortality rate and can lead to significant levels of neurological morbidity. Magnetic resonance imaging offers the potential to diagnose intracranial tuberculoma and to differentiate it from other single hyper dense intracranial lesions.

Methods: The authors report thirteen patients who proved to have intracranial tuberculoma with emphasis on their magnetic resonance imaging. They all presented with varying degrees of raised intracranial pressure. All the thirteen patients had a CT scan which showed a single hyper dense lesion. After contrast the density of the lesions increased. To confirm the diagnosis of intracranial tuberculomas and to differentiate it from intracranial single hyper dense lesions, conventional pre and post contrast MRI imagining followed by FLAIR sequences and diffusionweighted echo-planner imagining (EPI) was carried out.

Results: All our patients were treated by corticosteroids for eight weeks and anti-tuberculous medication for a minimum period of twelve months. The treatment was successful in all our patients and none required surgery.

Conclusions: Early and clear diagnosis of intracranial tuberculoma can be achieved by MRI imaging including flair sequences and diffusion-weighted Echo-planner imaging, (EPI). This leads to early treatment and better outcomes of tuberculosis of the central nervous system.

Trial registration: This study was not registered as a trial because it is a retrospective study that does not include any involvement in the management or follow-up of patients and as such did not require registering.
\end{abstract}

Keywords: Tuberculoma, Magnetic resonance imaging, Flair sequences, Spectroscopy

\section{Background}

Tuberculosis of the central nervous system, caused by Mycobacterium tuberculosis, is more common among children, and it accounts for around $1 \%$ of all cases of tuberculosis [1], previously reported only in developing countries and among human immunodeficiency virus-positive migrants in developed countries. Recently, there has been resurgence of the disease in both the developing and the developed world [2]. This could well be due to the recent advances in different methods of magnetic resonance imaging. It used to carry a high mortality and a distressing level of neurological morbidity.

\footnotetext{
* Correspondence: ahmad_a_moussa@yahoo.com

${ }^{1}$ Department of Neurosurgery, Assiut University Hospital, Assiut, Egypt

${ }^{2}$ Department of Neurosurgery, University Hospitals Birmingham, Birmingham,

England

Full list of author information is available at the end of the article
}

Commonly, it presents as tubercular meningitis or post-tubercular meningitis hydrocephalus. Sometimes, it presents as a hyper dense space occupying lesion, usually in adults, known as tuberculoma.

The first differential diagnosis used to be secondary tumour in the brain, even when there is no known primary tumour.

Now, with recent advances of magnetic resonance imaging, it has become easier to diagnose intracranial tuberculoma and to differentiate it from other single hyper dense intracranial lesions. This has led to an earlier and successful medical treatment of the disease.

Neurosurgical intervention is confined to cases of hydrocephalus after tuberculous meningitis and rarely to large tuberculomas with space occupying effects. 


\section{Results}

Slow but progressive improvement has been demonstrated in all patients, both clinically and radiologically. On 6 and 12 months follow-up MRI scans, the lesions became smaller in size and less dense. After 24 months of treatment, the MRI demonstrated no enhanced lesions. There were areas of calcification in some patients which meant total resolution of the tuberculoma.

\section{Discussion}

Central nervous system tuberculosis remains a clinical challenge. It is rare and has no specific symptoms. It used to be difficult to differentiate between patients with intracranial tuberculoma and patients with neoplastic lesions.

Its pathogenesis is still incompletely understood. The most common view is that the bacilli reach the central nervous system by a haematogenous route secondary to tuberculosis elsewhere in the body. The original description by Rich and McCordock [3] suggested that tuberculous lesions develop in the brain during the stage of bacteremia. Later, one or more of these lesions rupture producing CSF tuberculosis. The initial focus may occur in the meninges, subpia or subependymal surface of the brain. The growth of these lesions is known to be immunological in nature [4].

Tuberculomas are firm, avascular, spherical masses with diameters varying between 2 and $10 \mathrm{~cm}$. They are well circumscribed, and the compressed surrounding brain tissue shows oedema and gliosis. The inside of the masses contains areas of necrotic caseation in which tubercle bacilli may be found [5].

It often presents with symptoms and signs of focal neurological deficit without systemic disease evidence. The radiological features, until recently, were also non-specific, and malignant lesions were the most acceptable differential diagnosis.

The recent diagnosis of intracranial tuberculoma is mainly based on the new development of the magnetic resonance imaging. These new developments are fluid-attenuated inversion recovery (FLAIR sequences) of the brain and the diffusion-weighted echo-planar imaging (EPI).

Following the diagnosis, anti-tuberculous treatment started. The results of this treatment are indirectly confirming the diagnosis.

We look forward to seeing other publications on the diagnosis of solitary hyper dense intracranial lesions.

\section{Conclusions}

Intracranial tuberculoma presents as a single hyper dense intracranial lesion.

Early and clear diagnosis of intracranial tuberculoma can be achieved by MRI imaging including flair sequences and diffusion-weighted Echo-planar imaging (EPI).
This is expected to lead to early treatment and better outcome of tuberculosis of the central nervous system. It will become less frequent and have minimal mortality and morbidity.

\section{Methods}

This is a retrospective analysis of 13 consecutive patients who presented to the Department of Neurosurgery at Assiut University Hospital, Egypt, and proved to have intracranial tuberculomas.

The inclusive criteria were manifestations of raised intracranial pressure and the presence of a solitary hyper dense intracranial lesion on CT or MRI.

The clinical presentation, radiological investigations and treatment of our patients are presented.

They were ten males and three females. The youngest was a boy of 10 years, and the other patients were adults with ages ranging from 40 to 60 years. Only two of the 13 patients became known, after presentation, to have tuberculosis. The others were generally healthy people with no past history of tuberculous or any other debilitating or specific disease.

At an earlier stage, when we were highly suspicious of the nature of the lesion and in spite of the MRI findings, two of our patients had CT-guided stereotactic biopsy using the CRW frame. The histopathology of these patients came as tuberculoma.

The two patients who were known to have tuberculosis had discitis at lumbar 3/4 and 4/5 levels, and each had a large psoas cold abscess.

Four patients presented with late onset epilepsy.

Five patients presented with raised intracranial pressure and left-sided weakness. The 10-year-old boy and one adult patient displayed manifestations of generalised raised intracranial pressure only.

\section{MRI methodology}

In all our patients, MRI scans of the brain were performed using a 1.5 Tesla scanner with an eight-channel head coil. Conventional imaging included standard T1- and T2-weighted sequences, as well as contrast-enhanced T1-weighted and fluid-attenuated inversion recovery (FLAIR) sequences of the brain. We also performed diffusion-weighted echo-planar imaging (EPI) in the axial plane with a single-shot EPI-spin-echo (SE) pulse sequence with a diffusion sensitivity of $b=0$ and 1000 $\mathrm{s} / \mathrm{mm}^{2}$. Single voxel $1 \mathrm{H}$-MRS (SVS) was performed using a Point Resolved Excitation (PRESS) sequence prior to the contrast-enhanced sequence.

\section{MRI imaging findings}

In all patients, an intra-axial lesion was identified. In six patients, the lesion was found in the deep white matter, in one patient it was found in the left basal ganglia and 


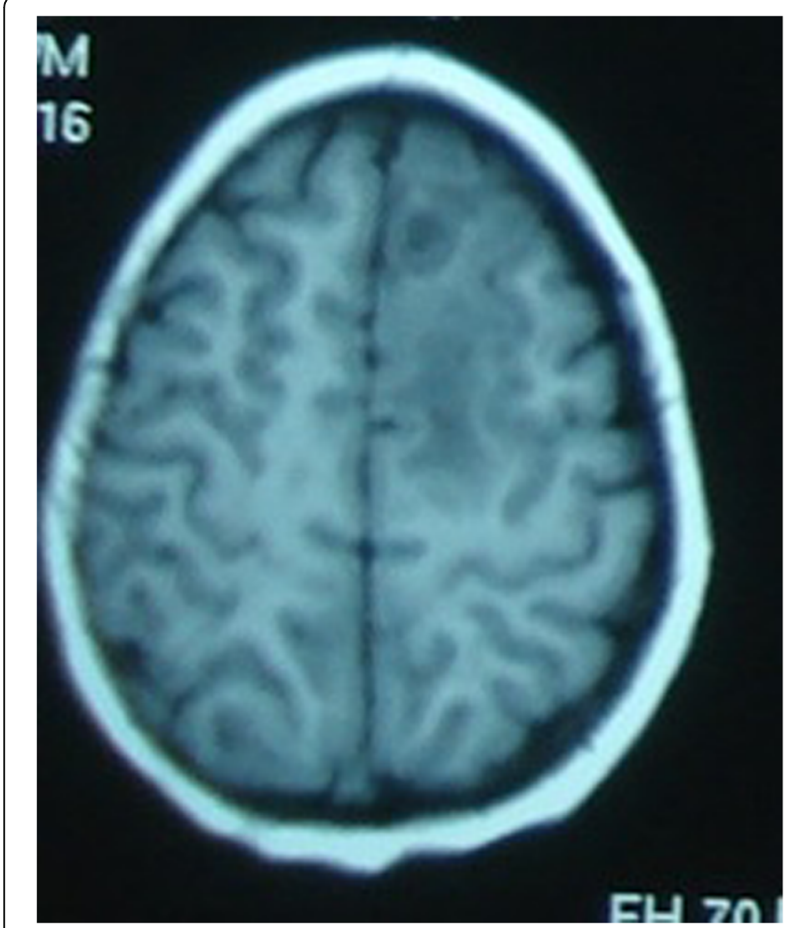

Fig. 1 Axial T1W showing a hypotense lesion with a relatively isointense rim

in six patients the lesion was found in the subcortical white matter. The lesion size ranged from 1 to $6 \mathrm{~cm}$. The morphologic features of the lesions were analysed on T2-weighted images on the basis of their outer margins and the presence of projections from the wall. The outer margins of the lesions were smooth in six lesions, lobulated in three lesions and crenated in four lesions.

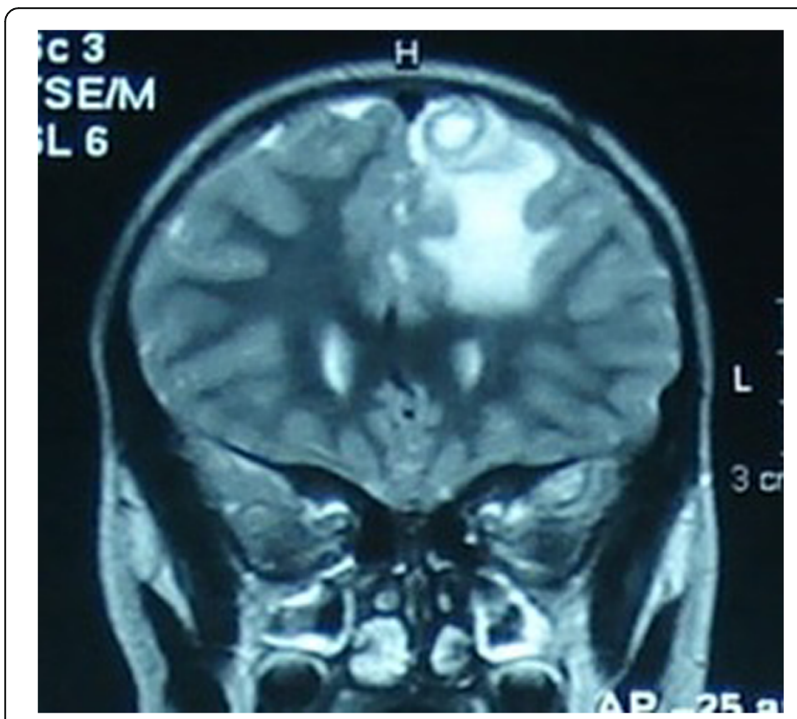

Fig. 2 Coronal T2W showing a hyperintense centre of the lesion with a peripheral hypointense rim and perilesional vasogenic oedema

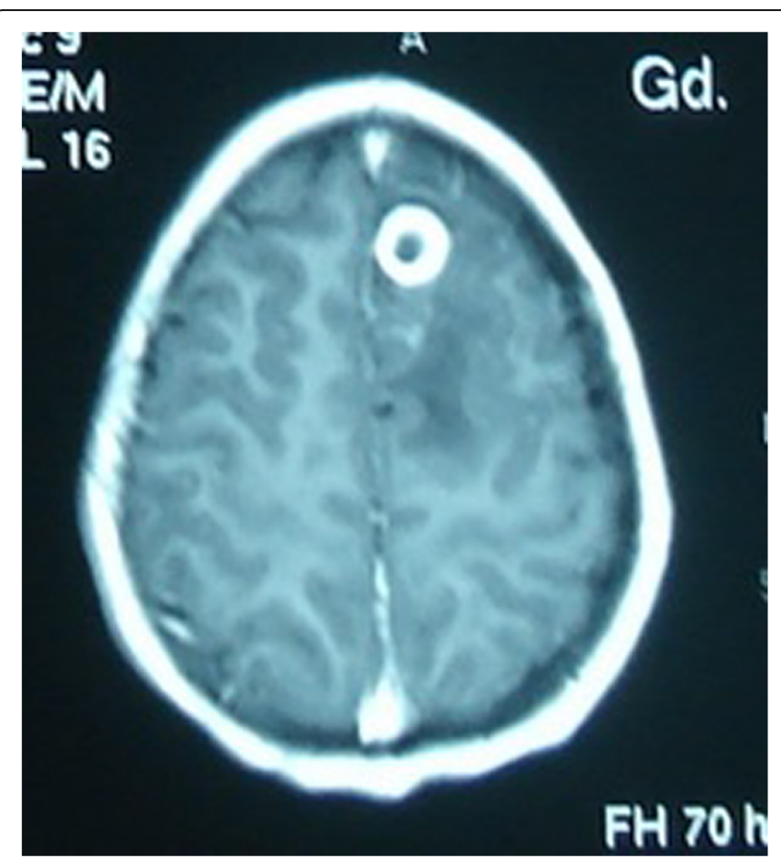

Fig. 3 Axial post-contrast T1W showing a hypointense lesion with a thick, smooth, intense rim enhancement

All the lesions were surrounded with vasogenic oedema. The T1W signal of all lesions was lower than that of the surrounding brain. In T2W images, six lesions showed hyperintense centres with a low-signal intensity rim while other seven lesions showed hypointense centres in

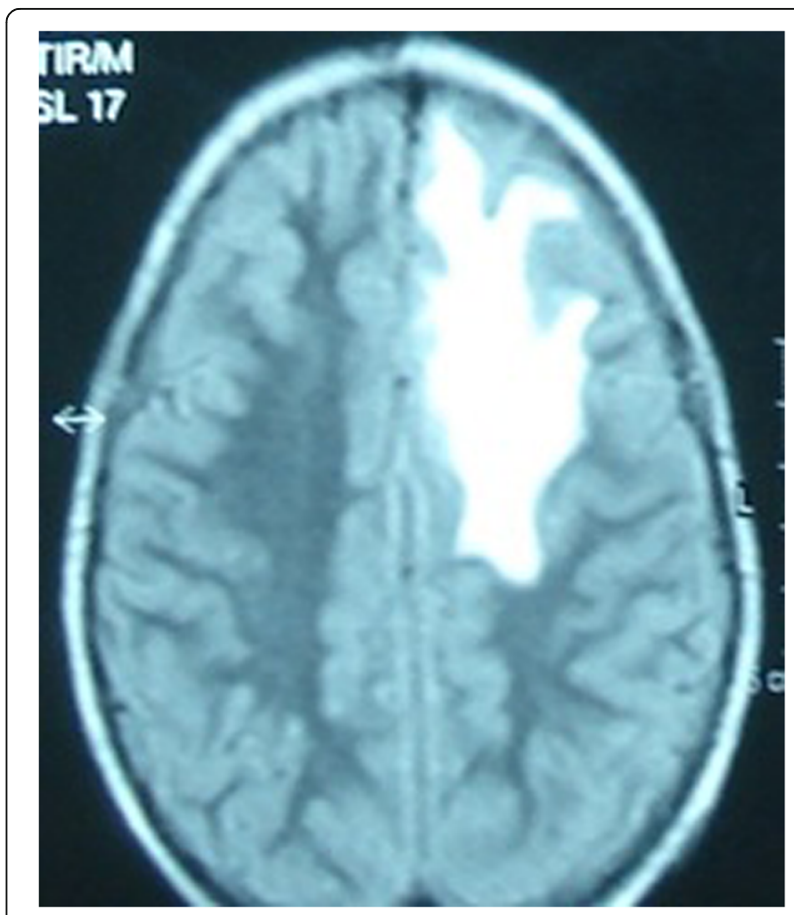

Fig. 4 Axial FLAIR showing perilesional vasogenic oedema that is disproportionate to the size of the lesion 


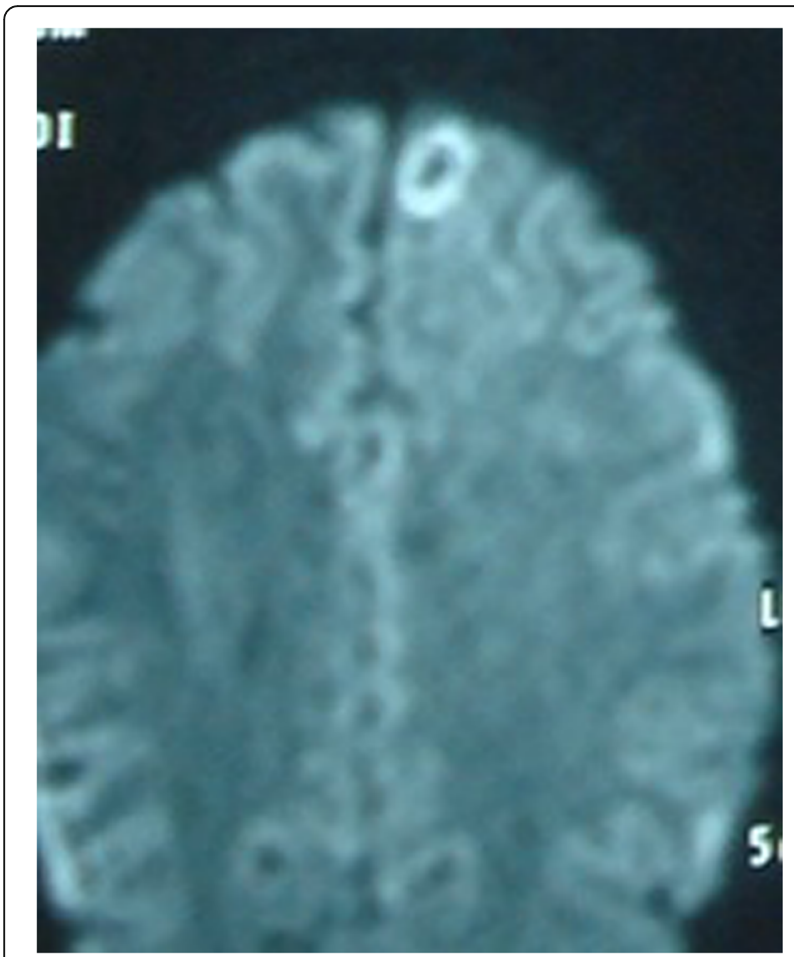

Fig. 5 Axial DWI showing a thick rim of diffusion restriction with central hypointensity (unimpeded diffusion)

three lesions and heterogeneous centres in four lesions. Following contrast injection, all the lesions showed a peripheral ring pattern of enhancement. In DWI, the lesions with hyperintense $\mathrm{T} 2 \mathrm{~W}$ centres showed a high-signal intensity diffusion restriction while the lesions with a T2W hypointense centre showed low-signal intensity. In MRS of all lesions, a diminished NAA peak and a prominently

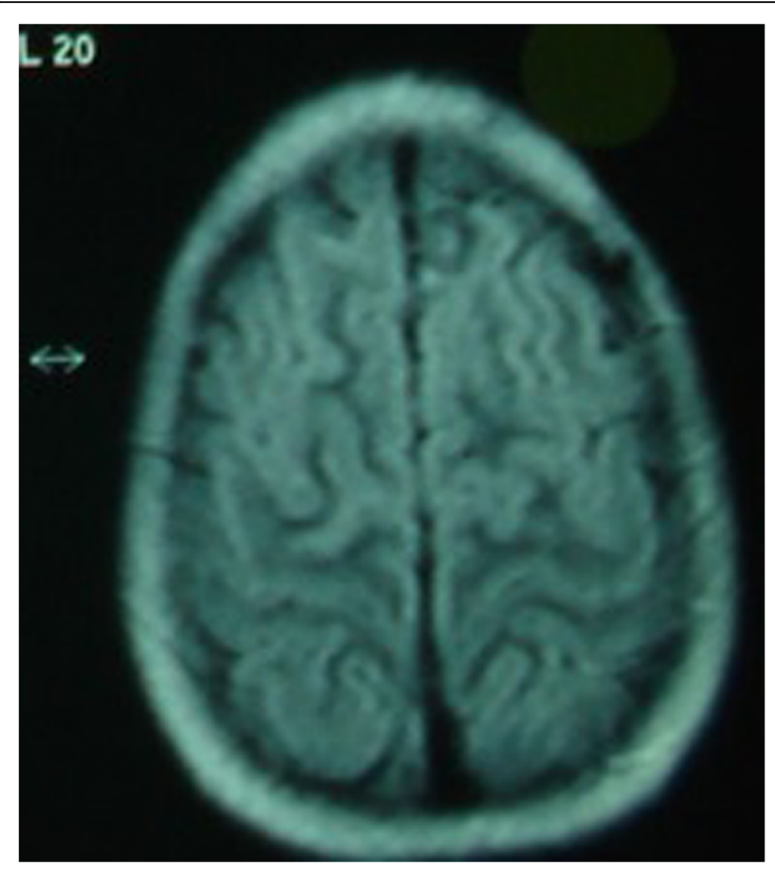

Fig. 7 Six months post-treatment axial FLAIR scan showing resolution of the perilesional vasogenic oedema with significant (more than 50\%) reduction of the size of the lesion

high lipid peak at $1.3 \mathrm{ppm}$ was evident. Lesions with a $\mathrm{T} 2 \mathrm{~W}$ hypointense or heterogeneous appearance showed Cho at $3.22 \mathrm{ppm}$ as well as lipids with associated increase in choline/creatine ratio greater than 1 (Figs. 1, 2, 3, 4, 5, 6 , and 7).

On T2 weighted images, the inner hyperintense rim corresponds to collagen fibres. The other partial or complete outer hyperintense rim corresponds to an inflammatory cellular infiltration surrounding the first rim.

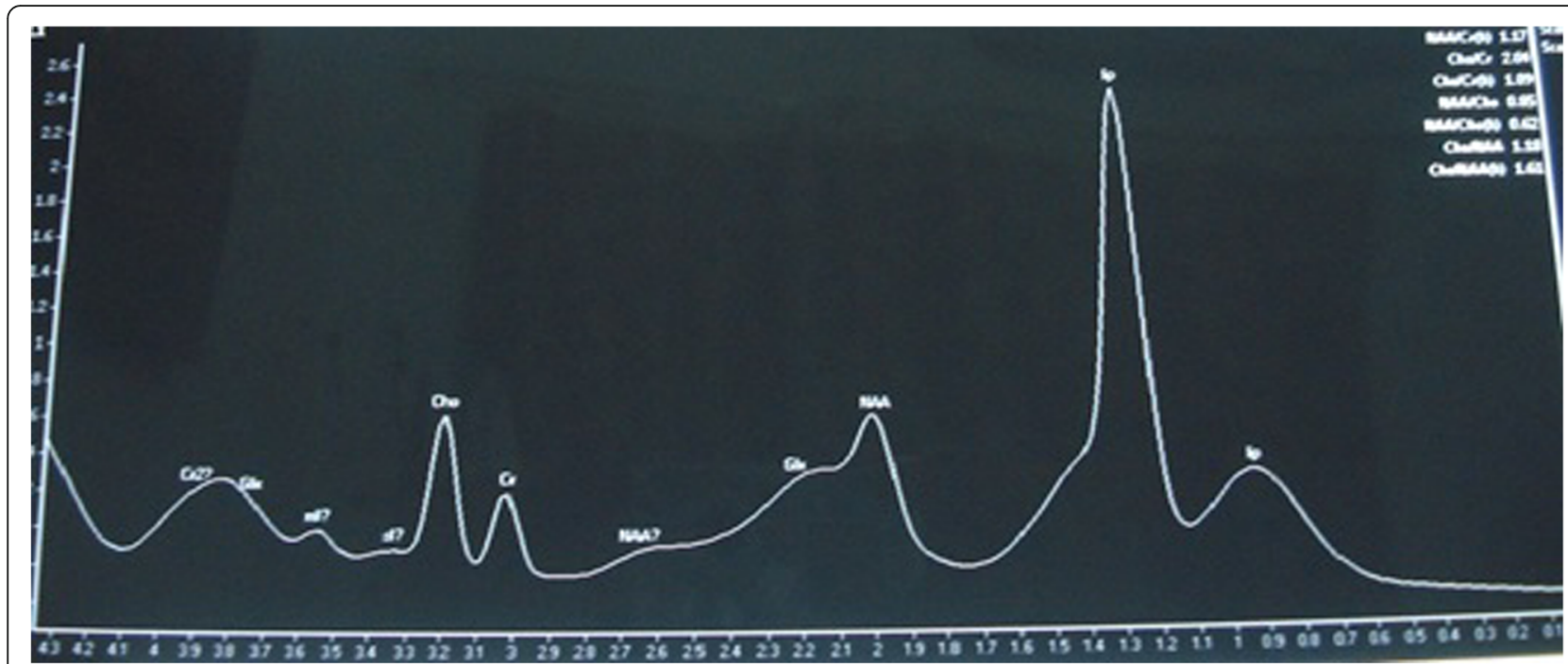

Fig. 6 Single-voxel spectroscopy obtained from the centre of the lesion showing markedly elevated lipid peak with depleted NAA, choline and creatine peaks 
Table 1 Summary of signal characteristics of individual lesions in different pulse sequences in our patients

\begin{tabular}{lllll}
\hline T1W & T2W & DWI & H1 spectroscopy & Number of lesions \\
\hline Low signal & High signal & High signal & High lipid peak & 6 \\
Low signal & Low signal & Low signal & High lipid peak + choline peak & 3 \\
Low signal & Heterogeneous & Heterogeneous & High lipid peak + choline peak & 4 \\
\hline
\end{tabular}

DWI diffusion weighted, T1W T1 weighted, T2W T2 weighted

A central isointense zone corresponds to the central caseation of the region.

On post-contrast $\mathrm{T} 1$ weighted images, there were single or multiple conglomerate ring enhancements within the tuberculomas in all patients, corresponding to both collagenous and inflammatory layers.

On T2-weighted images, the entire portion of the granuloma showed a heterogeneous isointensity or hypointensity with small markedly hypointense foci.

In summary, combination of the above-described signal intensity patterns and conglomerate ring like enhancing appearance of the lesions, in addition to the characteristic DWI signal intensity and the metabolic profile in $\mathrm{H} 1$ spectroscopy, are specific for tuberculoma. Also, these characters are important in differentiating intracranial tuberculoma from other ring enhancing brain lesions (Table 1).

\section{Treatment}

All our patients started corticosteroids (prednisolone $1 \mathrm{mg} / \mathrm{kg}$ ) and anti-tuberculous medication after admission as they all had raised intracranial pressure and their MRI showed cerebral oedema. The corticosteroids were given for 8 weeks.

The anti-tuberculous treatment was given following the recommendation of the Centre for Disease Control and Prevention [6].

The duration of anti-tuberculous treatment was based on the radiological responses of the tuberculomas [7].

\section{Acknowledgements}

The authors thank Rosemary Collier who provided medical writing support for the authors.

\section{Research design}

This is a retrospective study analysing historical data from patients' records and scans that were carried out at Assiut University Hospital.

\section{Authors' contributions}

AAM is the main author who contributed to the study design and writing of the publication. MEM contributed to the data collection. HAY contributed to the data collection, analysis of patients' scans and writing of the publication. All authors read and approved the final manuscript.

\section{Ethics approval and consent to participate}

Research committee approval was not required as it is a retrospective study with no identifying information of any patients. This study was not registered as a trial because it is a retrospective study that does include any involvement in the management or follow-up of patients and as such did not require registering.
Consent for publication

Not applicable.

\section{Competing interests}

The authors declare that they have no competing interests.

\section{Publisher's Note}

Springer Nature remains neutral with regard to jurisdictional claims in published maps and institutional affiliations.

\section{Author details}

'Department of Neurosurgery, Assiut University Hospital, Assiut, Egypt.

${ }^{2}$ Department of Neurosurgery, University Hospitals Birmingham, Birmingham, England. ${ }^{3}$ Department of Radiology, Assiut University Hospital, Assiut, Egypt.

Received: 15 January 2018 Accepted: 3 May 2018

Published online: 01 August 2018

\section{References}

1. Pimentel MLV, Alves SMV, Novis SAP, Brandoa RZ, Neto EB. Intracranial tuberculomas developing during treatment of pulmonary tuberculosis: case report. Arq Neuropsiquiatri. 2000;58(2-8):572-7.

2. Chaterjee SJ. Brain tuberculomas, tubercular meningitis, and post-tubercular hydrocephalus in children. Paediatr Neurosci. 2011;6(suppl1):S96-S100.

3. Rich AR, McCordock HA. The pathogenesis of tuberculous meningitis. Bull Johns Hopkins Hosp. 1933;52:5-37.

4. Bermudez LE, Sangari FJ. Cellular and molecular mechanisms of internalization of mycobacteria by host cells. Microbes Infect. 2001;3:37-42. (PubMed: 11226852)

5. Cherian A, Thomas SV. Central nervous system tuberculosis. Afr Health Sci. 2011;1:121-7.

6. Nahid et al. Official American Thoracic Society/Centers for Disease Control and Prevention/Infectious Diseases Society of America Clinical Practice Guidelines: Treatment of Drug-Susceptible Tuberculosis. Clin Infect Dis. 2016;32.

7. Poonnoose SI, Rajshekhar V. Rate of resolution of histological verified intracranial tuberculomas. Neurosurgery. 2003;53:873-9.

\section{Submit your manuscript to a SpringerOpen ${ }^{\mathcal{O}}$ journal and benefit from:}

- Convenient online submission

- Rigorous peer review

- Open access: articles freely available online

- High visibility within the field

- Retaining the copyright to your article

Submit your next manuscript at $>$ springeropen.com 